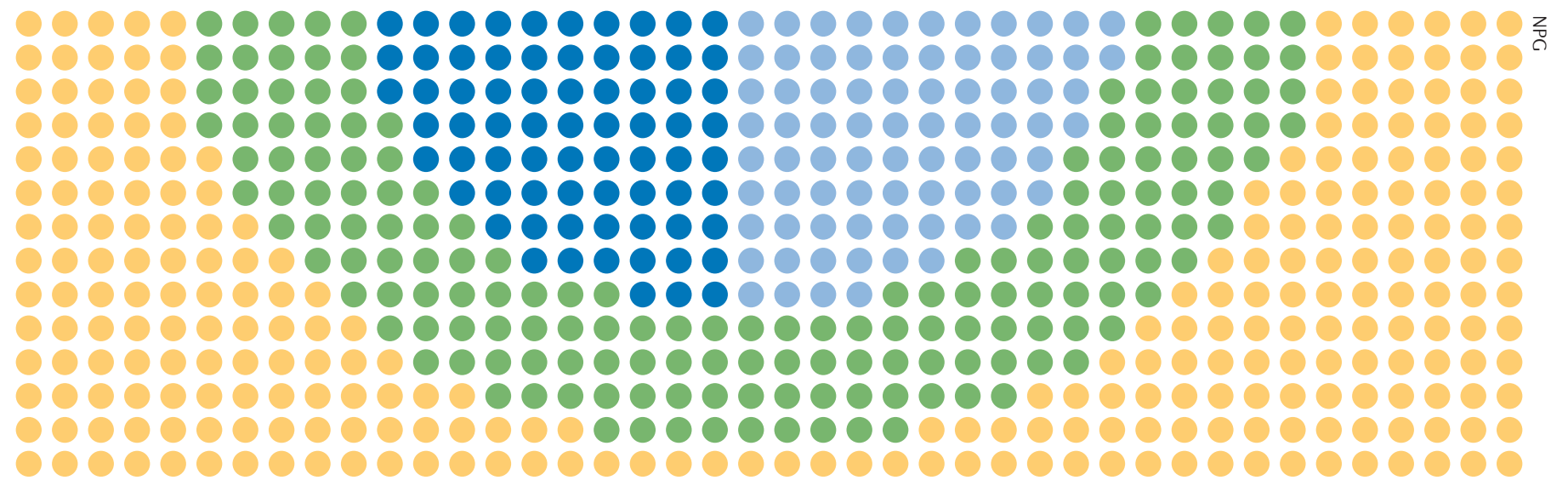

$\Rightarrow$ TUMOUR IMMUNOLOGY

\title{
MYCing around with the GC response
}

Germinal centres (GCs) are sites of B cell proliferation and antibody hypermutation. However, it has been unclear whether the cell cycle regulator MYC has a role in the GC response. Now, two independent studies describe a biphasic role for MYC in the formation and maintenance of GCs.

In response to a challenge with a T cell-dependent antigen, $B$ cells undergo rapid proliferation and form GCs. GCs are composed of two distinct areas: the dark zone, which is the site of somatic hypermutation; and the light zone, where $\mathrm{B}$ cells are selected to become memory B cells or plasma cells. Light-zone B cells can also re-enter the dark zone to undergo further proliferation and somatic hypermutation in a process termed 'cyclic re-entry'. Surprisingly, previous studies have shown that the expression of MYC is low or absent in bulk GC B cells, even though these cells are undergoing rapid proliferation. So, both groups sought to characterize MYC expression in B cells throughout the GC response.

The two groups used MYCreporter mice and found that MYC was expressed by a subset of lightzone B cells, but not by dark-zone $B$ cells. Furthermore, a population of $\mathrm{MYC}^{+} \mathrm{B}$ cells was identified early following antigen stimulation. Further studies by both groups revealed that at the initiation of GC formation, B cells expressed both MYC and B cell lymphoma 6 (BCL-6), a transcriptional repressor required for GC formation. However, as the GC developed, MYC expression was lost. MYC ${ }^{+} \mathrm{BCL}-6^{+} \mathrm{B}$ cells were hyperproliferative (Calado et al.), and BCL- 6 was shown to directly repress MYC expression in human GC B cells (Dominguez-Sola et al.). Through the conditional ablation of MYC, Calado et al. showed that MYC was absolutely required for induced and spontaneous GC formation. Together, these data suggest that early expression of MYC is required for the initiation of the GC response and that, as the response progresses, MYC expression is silenced by BCL-6 in dark-zone and most light-zone GC B cells.

Next, both groups analysed the small $\mathrm{MYC}^{+} \mathrm{B}$ cell population (which constituted $\sim 10 \%$ of the total B cell population) in mature GCs. Unlike MYC $^{-}$GC B cells, these light-zone $\mathrm{MYC}^{+} \mathrm{B}$ cells had a surface-molecule and gene-expression phenotype typical of recently activated lymphocytes, and a large fraction were actively proliferating. In addition, Dominguez-Sola et al. showed that the $\mathrm{B}$ cell receptor (BCR) repertoire of these cells was enriched for highaffinity receptors compared with that of $\mathrm{MYC}^{-} \mathrm{B}$ cells. The interaction of
B cells bearing high-affinity BCRs with T helper cells in the light zone results in the positive selection of these B cells, and Dominguez-Sola et al. showed that experimentally triggered positive selection induced the expression of MYC in light-zone B cells. Finally, both groups showed that MYC expression in mature B cells was required for the maintenance of the GC through cyclic re-entry.

Together, these studies show that MYC is expressed during the initial B cell population expansion that forms GCs, is downregulated in dark-zone B cells by BCL-6, and is re-expressed in a population of light-zone B cells that are selected for maintaining the GC response through cyclic re-entry. These data have important implications for our understanding of B cell lymphomagenesis, as chromosomal translocations involving MYC occur in almost all Burkitt's lymphomas and a fraction of diffuse large $B$ cell lymphomas.

ight-zone

$B$ cells that are selected for maintaining the GC response through cyclic re-entry

Olive Leavy

ORIGINAL RESEARCH PAPERS Calado, D. P. et al. The cell-cycle regulator c-Myc is essential for the formation and maintenance of germinal centers. Nature Immunol. 23 Sep 2012 (doi:10.1038/ni.2418)|Dominguez-Sola, D. et al. The proto-oncogene MYC is required for selection in the germinal center and cyclic reentry. Nature Immunol. 23 Sep 2012 (doi:10.1038/ni.2428) FURTHER READING McHeyzer-Williams, M. et al. Molecular programming of B cell memory. Nature Rev. Immunol. 12, 24-34 (2012) 